\title{
An Integrated Syndromic Surveillance System for Monitoring Scarlet Fever in Taiwan
}

\author{
Wan-Jen Wu*, Yu-Lun Liu, Hung-Wei Kuo, Wan-Ting Huang, Shiang-Lin Yang and Jen- \\ Hsiang Chuang
}

Epidemic Intelligence Center, Taiwan Centers for Disease Control, Taipei City, Taiwan

\section{Objective}

To develop an integrated syndromic surveillance system for timely monitoring and early detection of unusual situations of scarlet fever in Taiwan, since Hong Kong, being so close geographically to Taiwan, had an outbreak of scarlet fever in June 2011.

\section{Introduction}

Scarlet fever is a bacterial infection caused by group A streptococcus (GAS). The clinical symptoms are usually mild. Before October, 2007, case-based surveillance of scarlet fever was conducted through notifiable infectious diseases in Taiwan, but was removed later from the list of notifiable disease because of improved medical care capacities. In 2011, Hong Kong had encountered an outbreak of scarlet fever $(1,2)$. In response, Taiwan developed an integrated syndromic surveillance system using multiple data sources since July 2011.

\section{Methods}

More than $99 \%$ of the Taiwan population is covered by National Health Insurance. We first retrospectively evaluated claims data from the Bureau of National Health Insurance (BNHI) by comparing with notifiable diseases reporting data from Taiwan Centers for Disease Control (TCDC). The claims data included information on scarlet fever diagnosis (ICD-9-CM code 034.1), date of visits, location of hospitals and age of patients from outpatient (OPD), emergency room (ER) and hospital admissions. Daily aggregate data of scarlet fever visits or hospitalizations were prospectively collected from BNHI since July 2011 . Over $70 \%$ of the deaths in Taiwan are reported to the Office of Statistics of Department of Health electronically. We obtained daily data on electronic death certification data and used SAS Enterprise Guide 4.3 (SAS Institute Inc., Cary, NC, USA) for data management and analysis. Deaths associated with scarlet fever or other GAS infections were identified by text mining from causes of death with keywords of traditional Chinese 'scarlet fever', 'group A streptococcus' or 'toxic shock syndrome' (3).

\section{Results}

From January 2006 to September 2007, the monthly OPD data with ICD-9-CM code 034.1 from BNHI showed strong correlation with TCDC's notifiable disease data $(\mathrm{r}=0.89, \mathrm{p}<0.0001)$. From July 6, 2008 (week 28) through July 28, 2012 (week 30), the average weekly numbers of scarlet fever visits to the OPD, ER and hospital admissions were 37 (range 11-70), 7 (range 0-20) and 3 (range 0-9). Eighty-five percent of the scarlet fever patients were less than 10 years old. In Taiwan, scarlet fever occurred year-round with seasonal peaks between May and July (Fig. 1). From January 2008 to July 2012, we identified 12 potential patients ( 9 males, age range $0-82$ years) who died of GAS infections. No report had listed 'scarlet fever' as cause of death during the study period.

\section{Conclusions}

Taiwan has established an integrated syndromic surveillance system to timely monitor scarlet fever and GAS infection associated mortalities since July 2011. Syndromic surveillance of scarlet fever through BNHI correlated with number of scarlet fever cases through notifiable disease reporting system. Text mining from cause of death with the used keywords may have low sensitivities to identify patients who died of GAS infection. In Taiwan, syndromic surveillance has also been applied to other diseases such as enterovirus, influenzalike illness, and acute diarrhea. Interagency collaborations add values to existing health data in the government and have strengthened TCDC's capacity of disease surveillance.

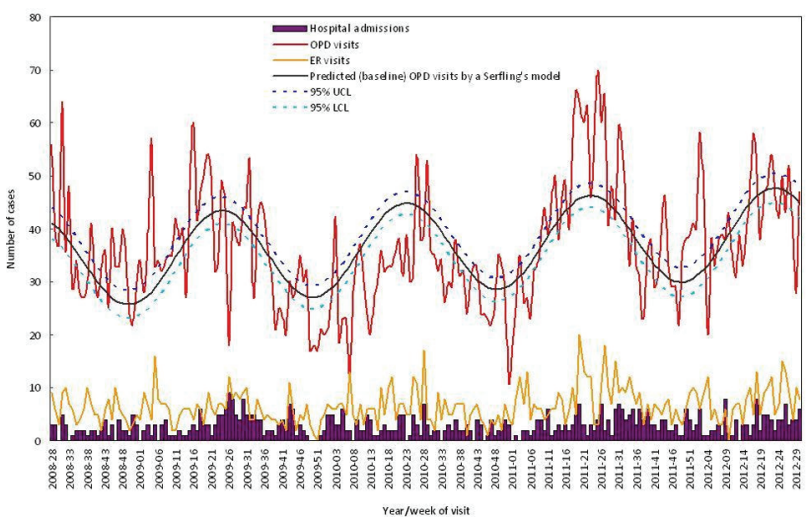

Fig. 1. Weekly numbers of nationwide scarlet fever OPD and ER visits, and hospital admissions, with baseline OPD visits and 95\% confidence interval calculated by a Serfling's model, week 28 of 2008 to week 30 of 2012 .

\section{Keywords}

syndromic surveillance; Taiwan; scarlet fever; claims data

\section{Acknowledgments}

We thank the Bureau of National Health Insurance and Office of Statistics of Department of Health for providing data required for this study.

\section{References}

1.Hsieh YC, Huang YC. Scarlet fever outbreak in Hong Kong, 2011. J Microbiol Immunol Infect. 2011;44:409-11.

2.Tse H, Bao JY, Davies MR, et al. Molecular characterization of the 2011 Hong Kong scarlet Fever outbreak. J Infect Dis. 2012;206:341-51.

3.Chen PJ, Wu WJ, Huang WT, Chuang JH. An early warning system for pneumonia and influenza mortality in Taiwan. Emerging Health Threats Journal 2011;4: 11024

\section{*Wan-Jen Wu}

E-mail: teatea@cdc.gov.tw 